gewarnt: "Einige der schwarzen nationalistischen Gruppen glauben, daß sie in

den Untergrund gehen müssen, weil sie angegriffen werden. Aber wir glauben nicht, daß man das Problem des Untergrunds romantisieren könnte. Dies ist eine prärevolutionäre Periode. Und wir fühlen, daß es notwendig ist, die Massen so lange zu erziehen, wie wir können.«

Detlev Claussen

\title{
Reform als Prozeß - \\ die Diskussion um die Bremer Juristenausbildung bis zur Universitätseröffnung *
}

\section{Vorbemerkungen}

I. Wer dachte, daß nach Verabschiedung der "Experimentierklausel « ${ }^{1}$ die seit Jahren in der Bundesrepublik geführte Diskussion um eine Reform der Juristenausbildung ${ }^{2}$ in ein neues Stadium treten würde, blieb bislang enttäuscht. Der Inflation der »Modelle $\ll^{3}$ im Zuge der Diskussion um die einphasige Ausbildung folgte bislang keine harte Währung der konkreten Vorschläge, der inhaltlich

\footnotetext{
* Anmerkung der Redaktion: Die folgende Dokumentation über den Planungsprozeß für die Reform des juristischen Studiums in Bremen soll beispielhaft zeigen, auf weldher Ebene und mit weldhen Fragestellungen heute die zuristische Ausbildungsreform diskutiert werden muß. Die Juristenausbildung in Bremen hat am 18. Oktober 197 I auf der Grundlage der Ankündigungen im Studienführer (Dokument s) begonnen. Ein repräsentativer Beridıt über das erste Semester ist für das nächste Heft zugesagt.

1 S sb und se des DRiG in der am 19. 9. I971 rom BT verabschiedeten Fassung. Vergl. den abweichenden Entwurf der BundesReg. in der BT-Drudksache VI/1 380 .

2 Vgl. die umfassenden Nachweise bei Bull, Zum Stand der Studienreform, JuS 69, S. 192 ff. sowie in den Gutachten $E$ und $\mathrm{F}$ zum 48 . Deutschen Juristentag, Walter Richter und Dietrich Oehler, In welcher Weise empfiehlt es sich, die Juristenausbildung zu reformieren?, Mündhen 1970 .

3 v. Brünnedk/Hein/Runge, Rahmenplan zur Reform der juristischen Ausbildung, Studentenvertretung der Juristischen Fakultät an der FU Berlin 9. Ir. I968;

"Bochum - Vorschläge zur Reform der juristischen Ausbildung, Arbeitskreis Juristenausbildung der juristischen Fachschaft an der Ruhr-Universität Bochum, SS 1969;

*CSU« - Münchner Modell für eine einstufige Juristenausbildung, 6. 4. 1970;

*CDU-Berlin - Vorschläge für eine Einheitsausbildung und deren praktische Durchführung in Berlin, 13. 3. 1970;

•Dekaner - Mainzer Beschlüsse zur Fortführung der Studienreform, vom I3/14. 2. 1970, z. T. in JuS 1970, S. 363 ff.;

* Hamburg « - Reformkommission für Juristenausbildung: Hamburger Modell einer einstufigen Juristenausbildung, Staatliche Pressestelle, I 5. 6. 1970;

Juristenverband, Deutscher -: Integrationsmodell (Bad Boll), 26. 6. 1970;

* Loccum - Entschließung des Loccumer Arbeitskreises für Juristenausbildung, in JuS 1970, S. SI ff.;

* Referendare I< - Reformkonzept des Bundesreferendarverbandes, in Recht und Politik 1969 Heft 4, S. 16 I ff.;

* Referendare II - Reformmodell des Südwestdeutschen Referendarverbandes (Stuttgart und Heidelberger Modelle) in Recht und Politik Heft 3, S. $108 \mathrm{ff}$;

*ASJ I - ASJ-Bundeskommission: Leitsätze zur Reform der Juristenausbildung, in Recht und Politik, Heft 2 1970, S. $4 \mathrm{I}$ ff.;

-SPD-Saar - Konzept zu einer Reform der juristischen Ausbildung, 25. Ir. 1969;
} 
und didaktisch durchstrukturierten Curricula, der politisch abgeleiteten und sich offen nach allen Seiten hin abgrenzenden Positionen. Die Diskussion um die konkret in Angriff genommenen einphasigen Studiengänge (z. B. Hamburg, Augsburg, Nordrhein-Westfalen, Bremen $)^{4}$ fand bislang hinter mehr oder weniger verschlossenen Türen statt. Der Grund liegt nicht nur in der Scheu aller Beteiligten, ihre Rolle im konkreten Reformverfahren durch den $»$ Schritt in die Offentlichkeit " zu durchbrechen, in ihrer Angst, Konflikte durch öffentliche Formulierung festzuschreiben, sondern vor allem darin, daß es nichts wirklich Neues, nichts wirklich Reformiertes zu veröffentlichen gibt. Die "Neue Juristenausbildung « $^{5}$ kommt eben nicht als Kind einer Reformkommission zur Welt, - gleichsam als geronnener kritischer Sachverstand aller Beteiligten. Wiethölter ${ }^{6}$ hat den Grund dafür ansatzweise angegeben: Reform der Juristenausbildung ist heute nur denkbar als Prozeß der reflektierten - von »einem transparent und plausibel gemachten gesellschaftspolitischen Engagement " getragenen - Destruktion der Ziele und Inhalte der alten Juristenausbildung, als Prozeß der "Entsperrung der heutigen institutionellen und psychologischen Lern- (und Lehr-) hindernisse ${ }^{7}{ }^{7}$ Eine solche Destruktion und Entsperrung ist aber nur praktisch möglich, ist nicht vorab konzipierbar als "Konkretisierung « eines Modells. Soweit die Diskussion um die Ausfüllung der Experimentierklausel diese Erkenntnis nicht zum Ausgangspunkt nimmt - und das ist erkennbar bislang außer in Bremen nirgendwo der Fall - muß sie notwendig hinter ihren eigenen Anspruch, Reform-Diskussion zu sein, zurüdkfallen. ${ }^{8}$

2. Ausgangspunkt aller Reformbemühungen in Bremen ist also: Es geht nicht um ein neues, ein "Bremer Modell" der Juristenausbildung, sondern um die Institutionalisierung von Bedingungen und Möglichkeiten einer praktischen Reform. Der eineinhalbjährige Diskussionsprozeß, der im folgenden in groben Zügen dokumentiert werden soll, ist nur zu verstehen als Auseinandersetzung über diese Bedingungen und Möglichkeiten. Die Dokumente sollen deshalb in drei Gruppen aufgegliedert und kommentiert werden: einmal als Ausdruck der Suche nach dem richtigen Bezugspunkt einer praktischen Reform ("Berufspraxis oder Wissenschaftskritik «), zum zweiten als Hypothesen über die Strukturerfordernisse eines umwälzenden Prozesses (»Integrierte oder spezialisierte Projekte und/oder Kurse? «), zum dritten als politische Positionen zum Problem des praktischen Verhältnisses eines Reformprozesses zu seinen Randbedingungen ("Taktische Anpassung oder permanente Revolte? «)

»ASJ II - ASJ WestlichesWestfahlen, Arbeitskreis Ausbildungsreform, Leitsätze zur Reform der Juristenausbildung, 28. 10. 1969, in Recht und Politik 1969, Heft 4, S. I62 ff.;

"Baukastenmodell - Groth, K., Juristisches Studium und Baukasten-Gesamthochschule - Ein Reformmodell in vier Phasen, in Weizädker u. a., Baukasten gegen Systemzwänge, München 1970; "Nordrhein-Westfalen « - Reform der Juristenausbildung II: Modell einer einstufigen Juristenausbildung, I9. 5. I971.

4 Für Hamburg und Augsburg vergl. Anm. 3, für NW ist ebenfalls wie für Bielefeld die Einführung noch unsicher, die in Anm. 3 aufgeführten Modelle sind da noch in der Diskussion, für Bremen vergl. die in der $\mathrm{KJ}$ 1970, Heft 3 S. 327 ff. veröffentlichten Arbeitspapiere zum sozialwissenschaftlichen Eingangsstudium, den Bericht über den Aufbau der Universität Bremen, Stand Dez. I970, sowie Universität Bremen, Studienführer und Personalverzeichnis, WS $71 / 72$.

5 Vgl. gleichnamiges Buch, herg. vom Loccumer Arbeitskreis, Neuwied und Berlin I970;

- Ebd., Didaktik der Rechtswissenschaft, S. 25 ff.

7 Ebd., S. 39 und 40 , in Klammern vom Verf.

8 Vgl. dazu Kröning, V., Konzepte der neuen Juristenausbildung, KJ 1970, Heft 3 S. 307 ff.

- Der Zusammenhang dieser drei Komplexe ist evident: Solange die Bezugspunkte eines Reformprozesses erst in diesem praktisch konkretisiert und revidiert werden können und müssen, gewinnt die Frage nach den Strukturen, die einerseits die Kontinuität des Prozesses, andererseits die ständige Revision und Neukonkretisierung ermöglichen, eminent an Bedeutung. Die 
3. Es kann im Rahmen einer Dokumentation über die Juristenausbildung nicht auf die Gesamtsituation der Bremer Universitätsgründung eingegangen werden, ${ }^{10}$ obwohl losgelöst von dieser Situation die Diskussion nur schwer verständlich ist. Einige kurze Thesen müssen als Orientierung ausreichen: Der historische Ort der Bremer Universitätsgründung mit ihren heutigen Intentionen liegt dort, wo reziprok zum bundesweiten Auflösungsprozeß der ehemals konsistenten sozialen Bewegung im Ausbildungsbereich ("Studentenrevolte«) der Staat den Zugriff auf diesen Bereich stückweise vorbereitet und strukturiert. Im kleinsten Bundesland Bremen gelingt es der »staatstragenden « SPD aus vielerlei Gründen ${ }^{11}$ nicht, den letzten Kraftakt der Studenten und Assistenten (politischideologische Sprengung des Killy-Gründungssenates) im Sinne dieses Trends mit der Institutionalisierung einer dem Staatszugriff ständig offenen Gründungsorganisation $\mathrm{zu}$ beantworten. Die im "Universitätserrichtungsgesetz « vorgesehene Organisationsstruktur ${ }^{12}$ entwidkelt eine Eigendynamik, die es einerseits ermöglicht, personell und informell einen Teil der im Zuge der Studentenrevolte erarbeiteten Diskussionsergebnisse und Problematisierungen in den Gründungsprozeß einzubringen, andererseits die sich formierende Reaktion aus dem Prozeß selbst ausschließt und auf eine Einflußnahme ${ }^{13}$ von außen (über Pressekampagnen und Senatspolitik) verweist. Der so einsetzende doppelläufige Diskussionsund Strukturbildungsprozeß (innerhalb der Gründungsgremien zwischen den einzelnen Linien des "Reformlagers«, zwischen Gründungsgremien und Umwelt über Legitimität und Funktionalität der beabsichtigten Reform überhaupt) wird mit beginnender Institutionalisierung der Universität selbst dann noch einmal überlagert von der Diskussion um Rechte und (in sich selbst natürlich wiederum höchst widersprüchliche) Interessen des Universitätssystems und der darin einbezogenen Personen. Die Diskussion um die Juristenausbildung war nun immer zugleich Teil dieses Kontextes wie auch Teil der allgemeinen Juristenausbildungsreformdiskussion, an der die Gründungsgremienmitglieder innerhalb ihrer Bezugsgruppen (hauptsächlich Frankfurt - die hessische Diskussion - und Berlin)

Diskussion solcher Strukturen ruft aber sofort den Gesetzgeber auf den Plan, der es für seine Aufgabe hält, Inhalte und Strukturen soweit wie möglich festzulegen, um damit eine rechesstaatliche Ausbildung zur Fähigkeit zum Richteramt zu sichern. Damit werden aber wiederum die Ausgangspunkte der Diskussion in Frage gestellt. In diesem Zirkel bewegte sich die Bremer Diskussion ständig; es wäre lohnenswert, die Frage zu untersuchen, in wieweit sie ihn arbeitsteilig bewältigt hat oder an ihm gescheitert ist.

$10 \mathrm{Vgl}$. hierzu folgendes (Reihenfolge chronologisch): $\gg$ Bremen zwischen Technokratie und Demokratisierung, * Albers/Behlmer/Loewe, studentische politik «, Bonn, April 1970, ebd. auch ausführliche Dokumentation des Zusammenbruchs des Killy-Senates; - Letzte Instanz: Verfassungsschutz - zur Ohnmacht der Reformer in Bremen , in sanalysen*, Nürnberg, Juni 1971; - Bremen - eine Standortfrage?*, W.-G. Brügmann, in scolloquium*, Berlin, Juli 1971; Lehrerbildung an der Uni Bremen*, bisher umfangreichste Dokumentation der Ereignisse bis zur Universitätseröffnung, in betrifft erziehung «, Weinheim, Berlin, Basel, Sep-

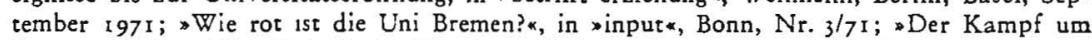
Bremens Universität«, Geis, I., in sHamburger Lehrerzeitung «, Nr. 15, Oktober 1971 .

11 Vgl. Bericht einiger Mitglieder der PKS der Uni Bremen«, Berlin 4. 7. 1970, unveröff. Manuskript, sowie Albers/Behlmer/Loewe, a. a. O. Anm. ro.

12 Einzig entscheidungsberechtigtes Organ ist ein ehrenamtlicher Gründungssenat, dem der Gesetzgeber keinerlei inhaltliche Auflagen machte und machen kann. Dieser schafft sich beratende Kommissionen für Sozialwissenschaften, Lehrerbildung, Naturwissenschaften, die ihrerseits zum einen Unterkommissionen bilden können, zum anderen hauptamtliche Planer wählen können, die das Land Bremen dann nach Gründungssenatsbeschluß einstellt. Zur Berufung der Hochschullehrer werden eigene Berufungskommissionen gebilder, die z. T. aus kooptierten außenstehenden Fachleuten bestehen.

13 Die Pressekampagnen sind $z$. T. dokumentiert in dem regelmäßig erscheinenden Pressespiegel der Bremer Universität, wegen der zahlreichen Bürgerschaftsdebatten vergl. Protokolle der Bremer Bürgerschaft seit Februar 1970, wegen der Senatseingriffe, vor allem in Personalentscheidungen vergl, die Dokumentation in sbetrifft erziehung*, a. a. O., Anm. IO. 
teilnahmen. Es kann nicht Aufgabe dieser Dokumentation sein, den Einfluß jeder dieser Variablen auf den Prozeß nachzuzeichnen und damit Hypothesen über die Möglichkeit und Fruchtbarkeit anderer in der Bundesrepublik anstehender Juristenausbildungsreformdiskussionen aufzustellen. Soldhe Hypothesen könnten jedoch Ergebnis weiterer an die Bremer Diskussion und die beginnende Realisierung anknüpfender Kontroversen sein.

\section{Bezugspunkt einer praktischen Reform: Berufspraxis oder Wissenschaftskritik?}

Die Diskussion um den Studiengang Rechtswissenschaft an der Universität Bremen begann im Frühjahr 1970 mit einer Kontroverse um das integrierte sozialwissenschaftliche Eingangsstudium (vgl. die in KJ 1970, S. 327 abgedrudkten Arbeitspapiere zum Sozialwissenschaftlichen Eingangsstudium). Zu dieser Zeit ging man noch davon aus, daß es möglich sei, die Inhalte der zu reformierenden Studiengänge und des Eingangsstudiums curricular durchstrukturiert festzulegen. Nachdem sich die Erkenntnis von der Unmöglichkeit eines solchen Vorgehens durchsetzte, begann die PKS und besonders die im Sommer gebildete Planungskommission Jura nach Bezugspunkten zu suchen, von denen aus es möglich sei, die herkömmlich gelehrte Rechtswissenschaft (sowie Ókonomie, Politologie, Soziologie und Geschichte) so kritisch umzustrukturieren, daß sie sich später im Ausbildungsprozeß selber zu neuen integrierten Curricula verdichten ließ. Dabei ging man davon aus, daß sich die Vermittlung in integrierten praxisorientierten Lehr- und Forschungseinheiten - in Projekten - vollziehen werde. Die Berliner Gruppe in der Unterkommission Jura/Planungskommission Sozialwissenschaften (UKJ/PKS) versuchte als diesen Bezugspunkt die Berufspraxis zu bestimmen und daraus Projektthemen abzuleiten (Dokument I).

Die Frankfurter Gruppe nahm diesen Ansatzpunkt auf, brachte jedoch von Anbeginn an einen mehr sozialwissenschaftlich fundierten rechtskritischen Aspekt mit ein (Dokument 2). Um zu beweisen, daß es möglich ist, vom Bezugspunkt juristischer Berufspraxisfelder aus Projektthemen anzugeben und zu konkretisieren, begann die UKJ die intensive Diskussion um Projektinhalte.

Man kam schließlich zu drei großen Bereichen, in denen Projekte anzusiedeln wären:

I. Kommunuale Organisation (Außendeterminanten der Arbeit in komplexen Organisationen),

2. Berufspraxis (Innendeterminanten des Verhaltens komplexer Organisationen)

3. Sanktion und Befriedung (Arbeits-, Sozial-, Miet-, Strafrecht und Resozialisation)

(Dokument 3). Im Zuge der Diskussion um Projektthemen zeigte sich eine doppelte Schwierigkeit: einmal war es unmöglich, Themen zu diskutieren ohne einen scharfen Begriff von dem, was Projekt eigentlich ist. Hier wurde also klar, daß die Konzeption der »Reform als Prozeß « nur durchführbar ist, wenn man präzise Vorstellungen über die Strukturen entwickelt, in denen sich dieser Prozeß abspielen soll (Dazu s. u. III. Abschnitt). Zum zweiten zeigte sich, daß die Isolierung von Projektthemen unter dem Bezugspunkt Berufspraxis selbst eine Aufgabe ist, die eines umfangreichen wissenschaftskritischen Vorlaufs bedarf, und endgültig erst im reformierten Studium selbst geleistet werden kann (vgl. Dokument 4). Unter immensem Zeitdruck (die PKS war monatelang ausschließlich 
mit Berufungsfragen befaßt) wurde im Sommer 197 I versucht, diese beiden Diskussionsprozesse (daß grundsätzlich ein Studiengang vom Problem der Berufspraxis her zu konstituieren ist, $\mathrm{da} ß$ es dazu aber eines wissenschaftskritischen Vorlaufs bedarf) mit der seit langem geführten Diskussion um das sozialwissenschaftliche Eingangsstudium zu verbinden und daraus das Modell des ab WS 1971/72 in Bremen anlaufenden integrierten Sozialwissenschaftlichen Eingangsstudiums zu entwickeln (vgl. Dokument 5 ).

\section{Strukturerfordernisse eines umwälzenden Prozesses: integrierte oder spezialisierte Projekte und/oder Kurse?}

Als zentrales wissenschaftspolitisches Problem der Bremer Universität kristallisierte sich im Laufe des Jahres 197 I das Problem des Unterrichts in Projekten und die dadurch bedingten Anforderungen an die Universitätsstruktur heraus. Die sich aus der vor allem von der Planungskommission Lehrerbildung vorangetriebenen Diskussion ergebenden Probleme wurden von der UKJ nur zögernd aufgegriffen. Sie stellten sich für den Bereich der Juristenausbildung auch besonders drastisch. Erst im Zuge der Diskussion konkreter Projekte gelang es, einigermaßen konkrete Vorstellungen von einem Jurastudium in Projekten zu entwickeln. Die Diskussion ist immer noch nicht abgeschlossen, da weder die endgültige Universitätsverfassung, noch ein Juristenausbildungsgesetz für Bremen bislang vorliegt.

\section{Verbältnis des Reformprozesses zu seinen Randbedingungen: taktische Anpassung oder permanente Revolte?}

Völlig ungeklärt war zu Beginn der Reformdiskussion das Verhältnis der Planungsgremien zu den außeruniversitären Stellen, insbesondere das Problem, wie inneruniversitäre Entscheidungen in ein vom Land zu erlassendes Juristenausbildungsgesetz mit entsprechenden Prüfungsbestimmungen zu transformieren seien. Erster Versuch, über dieses Verhältnis in die Diskussion zu kommen, waren die Thesen zur Bremer Juristenausbildung (Dokument 6). Der weitere Verlauf der Entwicklung ist dem Bericht über die Planung (Dokument 7) zu entnehmen. Die Diskussionen in der Universität liefen an verschiedenen Punkten immer wieder um das Problem, wie man sich staatlichen Interventionsversuchen gegenüber zu verhalten hätte. Als etwa die CDU in der Bürgerschaft den Antrag stellte, die Thesen zur Juristenausbildung, insbesondere These 2 Satz 2 dürfe der Bremer Senat bei seinen Entscheidungen über die Berufungsvorschläge der Bremer Universität nicht berücksichtigen, zerfiel die UKJ in zwei Fronten, von denen eine taktische Anpassung (= Zurüdkziehung der fraglichen These), die andere scharfe politische Stellungnahme forderte. Auch im weiteren konnte ein vollkommen durchkonzipiertes einheitliches Vorgehen in der Landeskommission nicht erreicht werden. Als günstig hat sich bislang ein Arrangement mit den »Praxisvertretern " der Kommission erwiesen, das auf langfristige gegenseitige Lernprozesse zielt unterstützt durch ständige wissenschaftliche Expertendiskussion über die in Bremen bereits ablaufenden oder demnächst vorgesehenen Studienphasen. Ein Ergebnis dieser Bemühungen und damit ein endgültiger Erfolg bei der Institutionalisierung der »Reform als Prozeß « ist bis heute nicht abzusehen. 
Diskussionsvorlage zur Frage des projektorientierten Studiums und der Bestimmung der Projektbereiche im Fachbereicb Sozialwissenschaft Bremen.

I. Gegenwärtig kann bei der Konzipierung einer sozialwissenschaftlichen Ausbildung nicht von einer Identität zwischen Theorie und Praxis ausgegangen werden. Diese könnte angesichts durch Kapitalverwertungsinteressen restringierter Möglichkeiten wissenschaftlich angeleiteter gesellschaftlicher Praxis nur um den Preis des Theorieverlusts angestrebt werden.

Soweit es für möglich und notwendig erachtet wird, eine Berufspraxis-Perspektive in das Studium der Sozialwissenschaften einzubeziehen, erscheint diese Perspektive notwendig getrennt von der Erarbeitung jener grundlegenden theoretischen Bestimmungen, die den Sozialwissenschaftler erst zur Erhellung konkreter gesellschaftlicher Praxis, auch seiner eigenen Berufspraxis, instand setzen kann.

2. Soll im Studium eine Berufspraxis konzipiert werden, in der wissenschaftlich angeleitete Praxis nicht von vornherein verkehrbar wird in ein bloßes Mittel zur erhöhten Ausbeutung der Lohnabhängigen, so müssen die Bereiche, in denen die stofflichen Fähigkeiten für eine sozialwissenschaftliche Berufspraxis erworben werden können, dahingehend ausgewählt werden, daß sie Kapitalverwertungsinteressen gegenüber neutral oder mindestens nicht direkt subsumiert sind.

[...]

3. Nach u. E. ist ein Bereich mit wachsenden Berufschancen für Sozialwissenschaftler, in dem sie exemplarisch die Qualifikationen für interdisziplinäre planerische Kooperation erwerben können, zu sehen im Bereich der sog. Infrastruktur. Dieser Bereich ist dadurch definiert, daß in ihm (unter den je spezifisch historischen Bedingungen) schwer oder überhaupt nicht Kapital verwertbar ist bzw. Arbeitskraft unmittelbar mehrwertbringend eingesetzt werden kann. (Was nichts damit zu tun hat, daß die in diesen Bereichen eingesetzten Arbeitskräfte ebenso oder noch schlechter als die direkt dem Kapital subsumierten Arbeitskrätte bezahlt werden können.) Andererseits gehört der Infrastrukturbereich - und zwar wachsend im Maße des technologischen Fortschritts kapitalistischer Produktion zu den allgemeinen Randbedingungen der Kapitalverwertung. $\mathrm{Da}$ das Kapitalverhältnis zusammenbrechen müßte, wenn die vom Kapital vernachlässigte sog. Infrastruktur nicht entsprechend weiterentwickelt würde, übernimmt der Staat in wachsendem Maße diese Aufgabe auf dem Wege der steuerlichen Umverteilung des gesellschaftlich produzierten Mehrwerts.

4. Innerhalb des Infrastrukturbereiches gibt es wiederum bestimmte Bereiche, die die Präferenz des Kapitals (bzw. bestimmter Kapitalgruppen, die Druck auf die jeweilige Regierung ausüben) haben und deshalb vordringlich oder umfassender als andere gefördert werden. Vor allem drei Bereiche können in dieser Weise als besonders kapitalnah bezeichnet werden:

Das Verkehrswesen (einschließlich Straßen-, Schienen-, Schiffahrts-, Luft-, Nachrichtenverkehr),

die Energie- und Wasserversorgung,

Forschung und Entwicklung (soweit sie in Produktionstechnologie umsetzbar ist).

Zumindest fünf Bereiche zeichnen sich demgegenüber $a b$, für die erst auf erhöhter Stufenleiter kapitalistischer Produktion öffentliches Problembewußtsein entsteht, und die in der BRD spezifisch vernachlässigt wurden in der verlängerten Nachkriegs-Rekonstruktionsperiode: 
Einrichtungen des Umweltschutzes (einschließlich Abwässer- und Müllbeseitigung, Reinhaltung von Luft- und Pflanzenwelt),

Einrichtungen der Städteplanung und der Planung des bäuerlichen Genossenschaftswesens,

Einrichtungen des Gesundheitswesens (einschließlich der Gesundheitsvorsorge und entsprechender Sozialbauten),

Einrichtungen des Freizeit-, Erholungs- und Sozialfürsorgebereiches (einschließlich Alters- und Jugendpflege),

Einrichtungen der Bildung und Qualifizierung der Arbeitskrätte (von der Vorschule über allgemeinbildende, berufsbildende und weiterqualifizierende Schulen bis zur Hochschule, einschließlich der unabtrennbaren Forschungspraxis).

5. Vor allem in diesen Bereichen scheinen uns Möglichkeiten für eine sozialwissenschaftlich-planerische Berufspraxis gegeben und die Konzipierung entsprechender Projekte in der sozialwissenschaftlichen Ausbildung (in Kooperation mit Naturwissenschaftlern, Stadtplanern, Landwirten, Medizinern, Architekten, Psychologen, Sozialarbeitern) geboten.

\section{DOKUMENT 2}

\section{Diskussionsbeitrag zur Vorbesprecbung am 26. 9. 1970}

Juridische Verkehrsformen erfordern Kenntnis und Gebrauch von Rechtstechniken vor allem in 3 Bereichen:

I. in der Justiz,

2. in sozialen Vertragsbeziehungen,

3. in der staatlichen Ordnungs-, Planungs- und Leistungsverwaltung

In diesen 3 Funktionen der Rechtsanwendung zielt die gängige juristische Praxis auf 3 Ziele: auf Stabilisierung des Status-quo, auf Legitimation der privatkapitalistischen Wirtschafts- und Sozialstruktur, auf Konzertierung des ungeregelten Wachstums. Allfällige Frontbegradigungen gegen einzelne Machtgruppen kommen hinzu. Bedürfnisse, die in den zentralen Strukturentscheidungen vernachlässigt werden und im öffentlichen Bewußtsein verschüttet sind, finden in die juristische Praxis keinen Eingang. Die juristische Ausbildung immunisiert die soziale Wahrnehmung gegen wrechtsfremde Erwägungen «. Die Juristen haben bisher mit Fleiß betrieben, die Ungleichheit rechtsfähig zu machen.

Die Justiz hauptsächlich hat die Fasade der konfliktfreien formierten Gesellschaft mitgestützt. Die Gerichte überziehen Prozesse, in denen soziale Konflikte ausgetragen werden, mit einem Netz von Verfahrensvorschriften, so daß sie als Kanäle politisch in Gang gesetzter Veränderungen untauglich werden. Rechtsformalismen stehen anstelle von Verfahren, die für die Rücknahme eingeführter Regelungen offen und der Einführung neuer Geltungsgründe zugänglich sind.

Die scheinbare Sachlichkeit und Interessenneutralität des Richters entschärfen soziale Konflikte durch symbolische Befriedung. Zumal in Beziehungen, die das Verhältnis sozialer Gruppen zueinander regeln, schreiben sie Lösungen vor und sanktionieren sie, die das ungleiche Stärkeverhältnis der Gruppen entlang der Klassenschranke wiedergeben.

Die Rechtsexperten im Dienst von Verbänden, der Wirtschaft oder privater Parteien wirken in Saldo zugunsten der privatkapitalistischen Interessen. Der Grund dafür ist nicht nur, daß Produzenten und Vermögende mehr und besseres Rechtswissen kaufen können als Konsumenten und Lohnabhängige. Hinzu 
kommt, daß in der juristischen Praxis die Indifferenz gegen den gesellschaftlichen Kontext ihrer Sachverhalte latent ist, von neuen Theoretikern sogar zum Prinzip erhoben ist. Wenn man Rechtsentscheide offen mit den Sacherwägungen in Verbindung brächte, an denen sie sich faktisch orientieren, träte im Streit der Sachargumente die Uberforderung der Justiz zutage. Die absolute, überpolitische Autorität des Rechts gälte nicht mehr. Eine ideologische Agentur wäre nicht mehr funktionsfähig, mit der soziale Konflikte nicht entschieden, sondern suspendiert werden.

Die Kritik der Scheinformalisierung ungleicher Chancen in geregelte Verhältnisse und der Personalisierung sozialer in individuelle Konflikte ist in der Juristenausbildung nicht zu erwarten, solange Juristen nur juristische Fächer studieren und die kritische Analyse Probleme mit Blick auf rechtliche Lösungen stellt. Unter diesen Bedingungen werden Anwälte soziale Konflikte absorbieren, statt sie zu formulieren, und Interessengegensätze blockieren, statt sie entscheidungsfähig an die Kontrahenten zurüdkverweisen. Die Verwaltungsjuristen wikkeln Planungs- und Leistungsentscheidungen nach einem Verfahrensritual ab, in das ungefiltert die Zielvorstellungen ihrer Abnehmer eingehen. Sie lassen sich Tempo und Inhalte der Planung und Zeitpunkt und Ausmaß der Leistung von den privatwirtschaftlichen Interessengruppen vorschreiben. (Bauplanung, Sozialfürsorge, Wirtschaftssteuerung sind Beispiele.) Die Ministerialjuristen sind auf Zulieferung aus den Planungsstäben des BDI usw. angewiesen, da sie weder Sachkenntnisse noch Zugang zu den nötigen Informationen besitzen. Da sie die zugelieferten Programme nur juridifizieren und auf Stromlinie bringen, aber nicht überprüfen können, sind radikale Alternativplanungen von ihnen nicht zu erwarten.

Nach den verschiedenen Erfordernissen der 3 Arbeitsfelder müssen unterschiedliche Kombinationen juristischer und anderer gesellschaftswissenschaftlicher Kurse eingerichtet werden.

Daraus sind folgende Diskussionsthesen abzuleiten:

I. Mit der einheitlichen Juristenausbildung, wie sie selbst die avanciertesten z. Z. diskutierten Reformmodelle (Loccumer Modell) noch anstreben, muß gebrochen werden.

2. Inhaltliche Ausbildungsziel muß die Grundlegung eines gewandelten Selbstverständnisses sein, nach dem soziale Konflikte nicht mehr rechtstechnisch befriedigt, sondern ausformuliert und an die Parteien zur Entscheidung zurïckverwiesen werden. Dazu gehört die Befähigung der Richter, die materiellen Entscheidungsprämissen auszuweisen und wo nötig als widersprüchlich zu konstatieren.

3. Anwälte müssen während des Studiums Gelegenheit haben, sich in die Sachproblematik spezieller Rechtsbereiche einzuarbeiten. Sie sollen zur Vorwegnahme neuer Lösungsmodelle soziologische oder wirtschaftspolitische Phantasie entwidkeln (Mietrecht, Minoritätenschutz, Produzentenhaftung etc). Sie müssen in der Ausbildung Gelegenheit haben, Theorien und Lösungsmodelle anderer sozialwissenschaftlicher Disziplinen kennenzulernen.

4. Planung auf Gesetzesgrundlage macht den Staat zum Regulativ der privatwirtschaftlichen Interessen. Planer sollen sich in der Rechtswelt auskennen, denn die standardisierten Verkehrsformen unter Ausblendung des Datenkranzes sind rational und effizient. An erster Steile aber sollen Planer Konzepte produzieren und Freiräume aktiver Einflußnahme schaffen.

5. Die soziale Kaste der Juristen muß aussterben. Rechtskenntnisse werden nur in interdisziplinären Studiengängen vermittelt. Die Ausbildung führt zur Teil- 
nahme an Projekten, in denen der Unterbau staatlich sanktionierter Normen und die sozialen Folgen der zur Wahl stehenden Rechtsentscheidungen deutlich werden.

\section{DOKUMENT $_{3}$}

Projekte im Bereich der Juristenausbildung

I. Projektbereich Kommunale Organisation (Außendeterminanten der Arbeit in komplexen Organisationen)

I. Leistungen

I.I Beteiligte Fachwissenschaften: Okonomie, Rechtswissenschaft, Soziologiel Politologie, Geschichte; hilfsweise: Medizin, Architektur, Erziehungswissenschaft etc.

I.2 Bestandteil möglicher Studiengänge für Juristen, Soziologen, Okonomen, Sozialarbeiter etc.

I.3 Qualifikation für alle Berufspositionen in Wirkungs- und Funktionsbereich kommunaler Organisationen: Kommunalverwaltung im engeren Sinne, Zweckverbände und sonstige übergreifende Gebiets- und Funktionskörperschaften, kommunale Wirtschaftseinheiten, der Kommune zugeordnete privatrechtlich oder "gemischterechtliche " Organisationen etc.

I.4 Für andere Berufspositionen: Orientierung über Funktion kommunaler Organisation in der Gesellschaft, ihrer Struktur und Wirkungsweise sowie ihre Verschränkung mit Betrieben, Verbänden, Parteien, staatlicher Verwaltung etc.

I.5 (ausgeführt nur für Juristen):

Vermittlung der für einen formellen Abschluß notwendigen Kenntnisse in

- Staatsrecht (teilweise)

- allgemeinem Verwaltungsrecht

- besonderem Verwaltungsrecht

- Verwaltungsprozeßrecht (teilweise)

- Steuerrecht

- "Wohnrecht« (Zusammenfassung aller privat- und öffentlichrechtlichen Probleme am Zusammenhang mit der Bereitstellung und Nutzung von Wohnraum)

- Sozialrecht (teilweise)

- sowie Verwaltungspraxis etc.

2. Organisation und Inhalte

2.I Grundeinheit des Projektbereichs sind die Projektgruppen. Sie konstituieren sich aufgrund von Projektthemen. Die Projektthemen werden jeweils aufgrund konkreter in der Realität auffindbare Konflikte formuliert und sollten nach Möglichkeit der Projektgruppe auch Praxis in diesem Konflikt erlauben, zumindest aber Kritik von Praxis zulassen. Das Ensemble der Projektthemen sollte nach Möglichkeit folgende Problembereiche abdecken:

2.I.I Stadtplanung, Städtebau, Städtesanierung (mit den Grundkategorien: Ókonomie, wirtschaftliche Interessen, Bedürfnisse der Bevölkerung, poli- 
tische Umsetzung, d.h. Planungsverfahren, kommunale Demokratie, Vollzug durch Verwaltung und Wirtschaftsträger).

2.I.2 Wohnen und Mieten. (Neben den Kategorien zu I. zusätzlich: Verkehrsformen, rechtliche Regelungen, politische Aktionsformen)

2.r.3 Verkehr (ebenfalls mit den Kategorien zu r.)

2.1.4 Kommunale Funktionen im Bildungssektor (neben den Kategorien zu I. besonders: Vorschulen, Schulen, Erwachsenenbildung, Freizeitgestaltung, Kultur; Verhältnis zu privatwirtschaftlichen Organisationen mit äquivalenten Funktionen)

2.1.5 Kommunales Gesundheitswesen

2.r.6 Kommunale Ordnungs- und Sozialfürsorgefunktionen

2.I.7 Kommunale Wirtschaftsförderung

2.1.8 Kommunale Gebiets- und Verwaltungsreformen...

2.2 Je nach Lage der Projektthemen werden übergreifende Gruppenverbände gebildet, die gemeinsame Themen bearbeiten, Strukturwissen umsetzen und Spezialprobleme delegieren. Grundsätzlich erfolgt auch eine Kommunikation über den ganzen Projektbereich hinweg, entweder in Form von Papieren oder Plenumsveranstaltungen.

2.3 Je nach Bedarf werden schriftlich oder in Vorträgen folgende Grundlagenprobleme vermittelt:

2.3.I Geschichte der kommunalen Organisationen

2.3.2 Funktion der Kommune im politischen System

2.3.3 Funktion kommunaler Maßnahmen im Kapitalverwertungskreislauf

2.4 Je nach Bedarf werden in Kursform folgende Spezialkenntnisse vermittelt:

(Liste wäre von den einzelnen Fachrichtungen nach ersten Erfahrungen mit dem Projekt zu erstellen).

3. Beispiele für Projekte

3.I Kommunaler Verteilungskonflikt

Einfluß von großindustriellen Interessen auf die Politik einer Stadt, insbesondere deren Verwaltung (Bereiche: Stadtplanung, Schulprobleme), zu zeigen etwa an den Beispielen Rüsselsheim, Leverkusen; es kooperieren: Politökonomen, Verwaltungsjuristen, Soziologen mit Schwerpunkt Politik, Organisationen/Verbände, Historiker u. a.

3.2 Kommunale Planung

Schulwesen, Wohnungsbau, Gesundheitswesen etc.; Diskussion von Beteiligungsmodellen vor dem Hintergrund barrierensetzender Kapitalinteressen; es kooperieren: Politökonomen, Betriebswirte, Verwaltungsjuristen, Organisationsfachleute, politische Soziologen u. a.

II. Projektbereich Berufspraxis (Innendeterminanten des Verhaltens komplexer Organisationen)

Unter I. werden wiederum die Leistungen des Projektbereiches beschrieben.

$[\ldots]$

2. Folgende Ansatzpunkte - mit heuristischem $Z$ weck gesammelt - stecken den Umriß der Problemzone "Berufspraxis in komplexen Situationen « ab:

2.I Auf dem Niveau individueller Berufsarbeit bietet das Stichwort $»$ Mitbestimmung am Arbeitsplatz« den Einstieg. 
- Rollentheoretisch sind Konflikte am Arbeitsplatz zu beschreiben. Fragen der Frauenerwerbstätigkeit im Tarifrecht und in der Praxis, der Vergütungskriterien bei älteren Arbeitnehmern sind Beispiele.

- Sozialpsychologisch ist die berufliche Sozialisation zu untersuchen. Fragen des Motivationsverlustes, der Stabilisierung und Anderung bestimmter Ansprüche und Bedürfnisse sind aufzuarbeiten und auf die Leistungsnormen und Laufbahnrechte bzw. den Anspruch auf Arbeitsplatzsicherheit zu beziehen.

2.2 Auf dem Niveau der Organisation ist ein guter Bezugspunkt das Problem der Zielfindung komplexer Organisationen.

- Strukturfunktional können Fragen nach der Rolle der technischen Intelligenz in Organisationsentscheidungen analysiert werden. Die Funktion der professionellen (wissenschaftlich-technischen) Orientierung bei Organisationszielkonflikten kann an Fällen der Arzneimittelforschung oder in der staatlichen Wirtschaftsförderung untersucht werden. Die Ziele des Unternehmensverfassungsrechts, des Dienstrechts sind zu untersuchen. Fragen der Verwaltungskontrolle, der Fehlerhaftung können auftreten.

- Modellanalytisch können Fragen der mikro- und makroökonomischen Rationalität der Unternehmenskonzentration behandelt werden und auf Probleme des Kartellrechts angewandt werden. Aktuelle Anlässe können aufgegriffen werden.

- In betriebswirtschaftlichen Fallstudien kann die Beziehung der eingesetzten Technologie auf die Organisation des Betriebsablaufs und des Design der Hierachie untersucht werden. Rechtspolitische Fragen des Laufbahnrechts und Mitbestimmungsmodelle in privaten und öffentlichen Organisationen ergeben sich.

2.3 Auf gesellschaftlichem Niveau sind Ausgangspunkt die Realisierungsbedingungen der gestaltenden Verwaltung in der gesellschaftlichen Umwelt ihrer Planmodelle. Wieweit sind die Binnenstrukturen der sozialen Organisationen reformbedürftig, um die Beschaffung der politischen Legimitation langfristiger Reformen überhaupt zuzulassen?

- Mit einer gesellschaftlichen (makrosoziologischen) Entwicklungstheorie ist das dem Wirtschaftsrecht implizite Modell des Unternehmenszweckes historisch-komperativ zu relativieren. Die rechtlichen Folgen in Modellen anderer (zusätzlicher) Unternehmenszwecke sind herauszuarbeiten.

- Systemanalysen innovativer Organisationen sind auszuwerten. Folgerungen für das Arbeits- und Betriebsverfassungsrecht ergeben sich.

- Das Legimitätsdefizit politischer Programme kann hypothetisch-deduktiv analysiert werden. Parteienrecht, Presserecht, Hochschulverfassung sind zu prüfen, inwieweit sie Legitimationen für öffentliche oder individuelle Interessen beschaffen.

III. Projektbereich Sanktion und Befriedung

A. I Arbeits- und Sozialrecht und Mietrecht

I.I Projektbeispiel zum Arbeitsrecht: Soziales Schutzrecht und soziale Minoritäten (Kündigungsschutzrecht und Gastarbeiter).

- Welches sind die Ursachen und Folgen der erhöhten Einstellung ausländischer Gastarbeiter? (Okonomische Dimension) 
- Welche innerbetrieblichen Funktionen haben ausländische Arbeiter? (z. B. Verrichtung unqualifizierter Arbeit, Senkung des Lohnniveaus, Sündenbockfunktion). In diesem Rahmen sind zu untersuchen: Vergleich der Kündigungsquote bei deutschen und ausländischen Arbeitern, Ursachen; welchen juridischen Repressionen sind Gastarbeiter ausgesetzt? (z. B. Kettenarbeitsverträge); wie ist das Verhalten des Betriebsrats in Bezug auf Kündigungen von Gastarbeitern?

- Welche Schwierigkeiten entstehen bei der gerichtlichen Vertretung von Gastarbeitern?

- Analyse von Kündigungsschutzprozessen: Andert sich richterliches Verhalten in Prozessen mit sozialen Minoritäten? Weist die individualarbeitsrechtliche Rechtsprechung auch bei sozialen Minoritäten fürsorgliche Züge auf?

- Reaktion des justitiellen Systems auf veränderte soziale Konstellationen (rechtssoziologische Dimension zur theoretischen Klärung der vorigen - eher empirisch angelegten - Untersuchungen).

I.2 Dieses Projekt soll vermitteln:

- Einsicht in das Verhältnis von Recht und sozialen Minoritäten

- Probleme des Individualarbeitsrechts und kollektiven Arbeitsrechts (Betriebsverfassungsgesetz)

- Fragestellung für eine sozialanwaltliche Prozeßstrategie

Durch Zusammenarbeit von: Juristen, Soziologen, Okonomen und Sozialpsychologen.

(Es folgt unter 2. ein ähnlich angelegtes und gegliedertes Projektbeispiel zum Sozialrecht und zur Sozialhilfe)

[...]

3.I Projektbeispiel zum Mietrecht: Gesellschaftlich diskrimnierte Personengruppen als Mieter.

- Inwieweit spiegelt die Entwicklung des Mietrechts Veränderungen in den sozialen und ökonomischen Konstellationen wieder?

- Welches ist die typische Wohnungsmarktsituation für gesellschaftlich diskriminierte Personengruppen? (z. B. unverheiratete Mütter mit unehelichen Kindern, kinderreiche Proletarierfamilien, Gastarbeiter etc.).

- Welche Repressions- und Ausbeutungsmöglichkeiten stehen dem Vermieter zur Verfügung, ohne Gefahr zu laufen, von Mietern, die der Personengruppe oben angehören, deswegen gerichtlich belangt zu werden?

- Welche Rolle spielen hierbei die Interessenverbände der Vermieter und Mieter (z. B. weitere Stärkung der Vermieterposition durch Formularmietverträge des Haus- und Grundbesitzervereins etc.)? Insbesondere sind die Aktivitäten des Mieterbundes geeignet, die Position des Mieters faktisch $\mathrm{zu}$ verbessern?

- Inwieweit kann das Mietrecht dem Mieter überhaupt Schutz bieten, wenn er sich entschließt, gegen schikanöse Hausordnungen, Mieterhöhungen, Kündigungen etc. sich vor Gericht zu wehren?

Welche Funktionen erfüllen in solchen Konfliktsfällen die Gerichte?

Welche Folgen hat die Inanspruchnahme der Gerichte für die weitere Wohnsituation der Kläger (z. B. erhöhte Repressionen durch Vermieter, Zunahme der gesellschaftlichen Diskriminierung, weitere Verschlechterung der Position auf dem Wohnungsmarkt)? 
- Wie verhält sich ein solches Mietrecht zum grundgesetzlichen Gebor der

3.2 Bietet für Juristen: Einsicht in relative Bedeutungslosigkeit justitieller Konfliktregelung, in Notwendigkeit kollektiver Organisation der Mieter; Fähigkeit Zielprojektionen für ein neues Mietrecht zu entwerfen; Fähigkeit, justitielle Konfliktregelung individueller Mietstreitigkeiten in die Strategie für eine Verbesserung der Gesamtsituation der Mieter einzubauen etc. Beteiligte Disziplinen: Soziologie, Okonomie, Bürgerliches Recht, Verfassungsrecht, Verfassungsgeschichte.

\section{B.2 Strafrecht und Resozialisation}

Die vorwiegend auf beratende Berufspositionen im Bereich des Strafrechts zugeschnittenen Projekte sollen offene Disziplinierungsfunktionen der Justiz deutlich machen - im Gegensatz etwa zu Projekten im Bereich Arbeits-, Miet- und Sozialrecht, wo es eher um die Einsicht in Verschleierung der Lage der Unterpriviligierten durch wie auch immer durchlöcherbare Schutzgaratien geht.

Die generelle Fragestellung zielt auf die gesamtgesellschaftlich vermittelten Zuschreibungsmechanismen für abweichendes kriminelles Verhalten. Die Untersuchung der zuschreibenden Funktion von Justiz und Polizei ist in überlappenden Projekten $z$ wischen schichtenspezifischer Sozialisation und Reproduk tion von Kriminalität in totalen Institutionen anzusiedeln.

I. Ablaufmodelle krimineller Entwicklung:

Dialektik von schichtenspezifischer Sozialisation und gesellschaftlicher $\mathrm{Zu}-$ schreibung krimineller Merkmale. Ungleichheit von Lebenschancen; Frage: wer wird krinimell? Wie sieht eine kriminelle Biographie aus?

Es kooperieren: Strafrecht, Kriminologie, Sozialpsychologie, Psychoanalyse, Schichtensoziologie, Okonomie.

2. Selektionsverfahren durch Strafverfolgungsorgane und Justiz. Subsumtion durch den Richter (den wert-freien Rechtsanwendungsautomaten) oder vorurteilsbeladene Entscheidung in asymetrischer Kommunikation. Das Verfahren als Vereinzelungsstrategie gegenüber dem Angeklagten. Welche Dimensionen der "Täterpersönlichkeit* werden durch polizeiliche oder gerichtliche Verfahren ausgeblendet?

Es kooperieren: Strafrecht, Strafprozeßrecht, Kriminologie, Sozialpsychologie, Kleingruppentheorie.

3. Totale Institutionen - Resozialisation

(gesellschaftlich bedingte Reproduktion von Kriminalität).

Fürsorgeerziehung, Jugendstrafanstalten, Gefängnisse

als komplementäre Institutionen.

Schuldprinzip und Rückfallquote.

Alternativmodelle von Resozialisation und deren gesellschaftlichen Bedingungen (historisch-komperativer Ansatz).

Es kooperieren: Strafrecht, Kriminologie, Sozialpsychologie, Organisationssoziologie, Geschichtswissenschaft. 
aus dem, Gutachten zum integrierten sozialwissenschaflichen Eingangsstudium an der Universität Bremen unter besonderer Berücksichtigung des Frankfurter Reformversuchs mit Studienanfängern an der juristischen Fakultät (Bechmann, Koch, Rottleuthner, Frankfurt, August 1971, S. 43 f.)

Wir sehen drei Momente der juristischen Praxis, in denen je schon sozialwissenschaftliche Gehalte impliziert sind:

r. Einmal ist die Urteilspraxis orientiert an Alltagstbeorien über typische Verhaltensweisen. K. D. Opp ${ }^{1}$ hat rekonstruiert, welche Annahmen einem strafrichterlichen Urteil zugrunde gelegen haben müssen; wie ein rechtlich relevantes Motiv erschlossen wird aus beobachtbaren Handlungen, der materiellen Lage, dem Verhalten im Prozeß, den Vorstrafen - und zwar durch Hypothesen, die allemal kaum überprüft sind und denen sozialwissenschaftlich überprüfte Hypothesen widersprechen. (Allerdings erklärt Opp nicht, warum sich Richter auf solch problematische Alltagstheorien stützen.)

2. Richter nehmen fortwährend Funktionsbestimmungen von sozialen Institutionen vor. In der Wettbewerbs-Rechtsprechung wird ein Vertrags- und Marktmodell zugrundegelegt, dessen (überholte) historische Geltungsbedingungen sich recht gut angeben lassen. Die neuere ökonomische Diskussion über den Wettbewerbsbegriff wird nicht verarbeitet. Bei der Einschätzung des Streiks geht das $B A G$ vom theoretisch umstrittenen »Maximumtheorem « aus, von einer Okonomie des großen Güterhaufens, der im Interesse aller liege. Aus dieser Unterstellung eines Gemeininteresses wird dann die soziale Schädlichkeit des Streiks abgeleitet, der nur »ultima ratio " sein dürfe.

3. Die Rechtswissenschaft verwendet auf allen Stufen - der Deutung und Anleitung der Rechtsanwendung und der Interpretation von sozialen Regelungsbereichen - Handlungsmodelle, die von den unterschiedlichen Ansätzen einer Theorie sozialen Handelns her kritisiert werden können. Diese bislang wenig beachteten Handlungskonzepte halten wir für ein zentrales Bindeglied von Rechtsund Sozialwissenschaften (These 2). In der Jurisprudenz werden sie relevant:

(I.) bei der Rekonstruktion von Motiven

a) bei der Aufbereitung von Sachverhalten im Rahmen zivilrechtlicher Relationen etwa. Historische Fälle werden dabei, durch Indizien und Zeugenaussagen gestützt, aufbereitet mit Hilfe der Unterstellung eines »vernünftigen * Motivationsgeflechtes. ("Was hätte wer vernünftigerweise getan? «) Hier gehen Annahmen über »typisches«, »gewöhnlich« zu erwartendes Verhalten ein;

b) bei der Aufklärung kriminellen Handelns wird die Existenz eines bewußten Motivs unterstellt ("Sie müssen sich doch etwas dabei gedacht haben! «), über das der Täter sich prinzipiell im klaren sein muß. Für den Schuldspruch genügt letztlich das - unterstellte - »Motiv «, die Gesetzesverletzung »gewollt « zu haben.

(2.) Bei der methodischen Anleitung richterlichen Handelns wird ein Modell supponiert, in dem sprachliche Symbole (Gesetze, Dogmatik, Methoden) als motivfähig erscheinen. Nur diese Annahme einer prinzipiell sprachlichen Motivation (auch eines sprachfähigen: explizierbaren »Vorverständnisses «) macht überhaupt

\footnotetext{
1 Karl-Dieter Opp, Zur Anwendbarkeit der Soziologie im Strafprozeß, in: Kritische Justiz I970, S. $383-398$.
} 
die juristische Beschäftigung sinnvoll. Denn nur auf diesem Hintergrund läßt es sich rechtfertigen, durch sprachliche Symbole Juristen zu sozialisieren, von Dogmatik eine voraussehbare, von Methodenlehren eine nachvollziehbare, von Prinzipien eine gerechte Urteilspraxis zu erwarten. Nur auf der Basis dieser empirischen Vermutung für ein hermeneutisches Motivationskonzept lassen sich symbolische Handlungsorientierungen aussprechen (These 3). Es ist aber die Frage, ob sich diese Hintergrundannahme selbst noch rechtfertigen läßt. Und an dieser Stelle treten die sozialwissenschaftlichen Handlungskonzepte in ein kritisches, weil konkurrierendes Verhältnis zu den juristischen (These 4).

Eine kritische Revision juristischer Motivzuschreibungen könnte etwa folgende Richtung nehmen².

I. a) Eine interaktionistisch (rollentheoretisch) geschärfte Analyse von Handlungsabläufen und der darin eingeschlossenen Rollenerwartungen könnte die bislang unkontrollierte Projektion von Alltagsvorstellungen ersetzen. Dabei zeigte sich auch, daß die Einschätzung von Motiven durchaus klassenspezifisch erfolgt. Der Vorwurf der Klassenjustiz zielt ja u. a. auf die ungleiche Verteilung von Plausibilitätschancen bei Sachverhaltsrekonstruktionen. Dem einen schenkt man Zutrauen, dem anderen traut man so manches zu.

b) Die Ergebnisse psychoanalytischer Kriminalitätstheorien werden zwar allmählich in der juristischen Kriminologie (Ätiologie und Resozialisierung) zur Kenntnis genommen. Aber die Konsequenzen des psychoanalytischen Ansatzes reichen bis in die strafrechtlichen Handlungstheorien. Zudem macht er die derzeitige prozessuale Motivzurechnung von Willensschuld durchsichrig als bloße Projektion repressiver sozialer Normalitätsanforderungen, unter die der Täter, der seine Motive im Rahmen der Prozeßkommunikation allenfalls als $»$ Rationalisierungen « zum Ausdruck bringen kann, subsumiert wird. Psychoanalyse führte nicht nur zur Revision dogmatischer Grundbegriffe (Handlung, Wille, Schuld, Strafe etc.); sie machte auch eine Umstrukturierung der Prozeßsituation notwendig; dabei könnten auch Analysen von verzerrten Interaktionen herangezogen werden. Sie löste schließlich nicht allein das symbolische Geflecht von Gewaltverhältnissen auf, sondern auch deren manifeste Architektur.

2. Interaktionsanalysen könnten den wie auch immer verzerrten kommunikativen Aspekt der Rechtsanwendung erhellen. Die monologisch vollziehbare Subsumtion erweist sich dabei allenfalls als der Endpunkt eines Verfahrens, in dem intersubjektiv argumentiert wird. - Eine allgemeine Kommunikationstheorie wäre in der Lage, den laxen Gebrauch des Vorverständnis-Begriffs zu disziplinieren. - Die "praktische Leistung der Rechtsdogmatik « (Wieacker), nämlich ihre (u. a.) handlungsorientierende Kraft, läßt sich vom Paradigma der Verhaltenswissenschaften her bestreiten. (In welche immanenten Schwierigkeiten ein solcher Ansatz auch immer bei der Analyse richterlichen Handelns gerät - das Paradigma hat einiges für sich.) Es sind dann nämlich weniger sprachliche Symbole, die richterliches Handeln steuern, sondern tiefsitzende Dispositionen, die familialen und sekundären Sozialisationsprozessen entstammen. Diese durch (un)lustvolle Stimulierungen gelernten Orientierungen legen den begrenzten Handlungsspielraum fest, in dem die symbolischen Elemente nur mehr eine Darstellungsfunk-

2 Dabei vertreten wir keinen Pluralismus von Theorien. Es lassen sich nämlich für bestimmte Problemstellungen Präferenzen für verschiedene Theorienansätze formulieren; es läßt sich sagen, an welchen Fragen sie scheitern. 
tion erfüllen. Zur Voraussage von Entscheidungen werden sie irrelevant. - Systemtheoretisch ließen sich Aussagen über die Bedingungen von Entscheidungen in formalen Organisationen treffen. Dann wird die juristische Symbolik durch Strukturen organisierter Verfahren abgelöst. Es wären Einschätzungen der sozialen Funktion von juristischen Entscheidungsinstanzen möglich, die über die professionellen, der Selbstverständigung dienenden Rechtfertigungen hinausgehen. - Ebenso ließe sich klassentheoretisch die repressive Funktion des Staatsapparates und der juristischen Stäbe kritisieren. Grundlegende rechtliche Kategorien (Privatautonomie, Eigentum, Gleichheit etc.) gerieten unter Ideologieverdacht. Die formalrechtliche Abstraktion von den Produktionsbedingungen läßt Ausbeutungsverhältnisse unberührt, legitimiert sie eher durch den Schein rechtlicher Gleichheit. Juristen erwiesen sich als in dogmatischer Selbsttäuschung befangen.

\section{DOKUMENT s}

Drei Veranstaltungen der Eingangsphase, aus dem I. Studienfübrer der Universität Bremen

S.I Sozialwissenschaftliche Berufsfelder

An der Planung beteiligte Hochschullehrer: Billerbeck, Hickel, Grauhan, Rinken, Lautmann (VAK 40)

Gegenstand der Berufsfeld-Veranstaltung ist die Analyse sozialwissenschaftlicher Berufstätigkeiten in den gesellschaftlichen Zusammenhängen, in denen sie erfolgen. Ziel dieser Analyse ist die Erlangung eines Bewußtseins von den objektiven gesellschaftlichen Funktionen von Sozialwissenschaftlern.

I. Die Berufsbilder der Studenten (behandelt anhand der zu Beginn des Semesters durchzuführenden Studentenbefragung);

- Problematisierung der Berufsbilder;

- Abhängigkeir der Berufsbilder vom Ausbildungsgang;

- Veränderung von Berufsbildern in Ausbildungsreform-Diskussionen.

II. Die tatsächlichen Berufsfelder von Sozialwissenschaftlern:

a) Faktische und antizipierende Funktionsbereiche (z. B.: Politische Bildung/politischer Journalismus; Verbands- und Parteiarbeit; Verwaltung: Planung/Entscheidung, Organisation/Personal; judizielles Entscheiden, judizielle Beratung: Entscheidungsinitiierung/Entscheidungsvermeidung; Sozialisation/Resozialisation; soziale Vorsorge: Gesundheitswesen, Sozialversicherung; ökonomische Globalplanung, Wirtschaftsberatung, Betriebsökonomie);

- Berufssoziologie;

- Sekundäre Sozialisation;

- Kontrolle - Herrschaftsausübung und soziale Stabilisierung;

- Soziale Lage (als Angehörige des unselbständigen Mittelstandes u. a.).

b) Prozesse der Veränderung der Berufsfelder im Kontext der Entwicklung der Bundesrepublik:

- Exkurs über die historische Entwicklung der Juristenberufe.

III. Sozialwissenschaftliche Berufspraxis innerhalb gesellschaftlicher Reformen: 
- Gesellschaftliche Reformerfordernisse und Reformbereiche;

- deren ökonomische und politische Bedingungen.

IV. Hochschul- und Ausbildungsreform als Reflex gesellschaftlicher Reformerfordernisse und Gegenstand sozialwissenschaftlicher Berufspraxis:

- Hochschulreform und Reform sozialwissenschaftlicher Studiengänge;

- Anderung gesellschaftlicher Anforderungen an die Qualifikation der menschlichen Arbeitskraft;

- Strukturveränderung von Institutionen und Apparaten (Insider- und Outsider-Strategien u. a.).

V. Der Doppelcharakter gesellschaftlicher Reformtätigkeit.

5.2 Struktur der bürgerlichen Gesellschaft

An der Planung beteiligte Hochschullehrer: Steinberg, Hahn, Jaroslawska, Rinken, Grauhan, Dubischar, Hinz, Biesecker, Wohlmuth, Saurien, Hickel, Stuby, Däubler, Preuß, Kade, Hoffmann, Sonnemann (VAK $4 \mathrm{I}$ )

I. Durchsetzung der bürgerlichen Gesellschaft, wie sie sich in ihrer Theorie darstellt.

II. Versuche zur Konstituierung bürgerlicher Freiheit:

a) unabhängig von feudalen Institutionen;

b) gegen feudale Institutionen.

III. Konstituierung der Lohnarbeit.

IV. Industrielle Revolution,

von der Manufaktur zur Großindustrie, Arbeiterbewegung, Arbeitergesetzgebung.

Fakultative Arbeitsgruppen:

Rolle des Staats-

$\begin{array}{lll}\text { apparates nach } & \text { Arbeiterbewegung } & \begin{array}{l}\text { Kritik der politischen } \\ \text { Oker industriellen }\end{array}\end{array}$ u.a.

Revolution

V. Formalisierung der Sozialwissenschaften.

Begründung:

Die Planung einer zentralen Veranstaltung über die Entstehungsbedingungen und die Entfaltung der bürgerlichen Gesellschaft leitet sich her aus der Kritik der heutigen Sozialwissenschaften. Kritisiert wird:

- Die Aufsplitterung in Einzelwissenschaften: damit Verlust der Möglichkeit gesamtgesellschaftlicher Theorie;

- Unhistorische Behandlung der Kategorien und des Gegenstandes der Sozialwissenschaften: dadurch Darstellung der Verhältnisse als nicht veränderbar;

- Mangelnder Praxisbezug der Ausbildung.

Daraus ergeben sich die Lernziele der Veranstaltung:

- Erkennen der Gesellschaft als gewordene und damit veränderbare;

- Kennenlernen der grundlegenden Kategorien zur Analyse der bürgerlichen Gesellschaft;

- Begreifenlernen, daß Theorien von gesellschaftichen Interessen abhängig sind, also nicht: dogmatisches Lernen von Theorien.

Erst wenn diese Lernziele ansatzweise erreicht sind und die Reflexion über Möglichkeiten sozialwissenschaftlicher Berufspraxis gemeinsam von Studenten und Hochschullehrern weitergetrieben wird, kann mit der Projektarbeit begonnen werden.

Methodisch und didaktisch liegt der Gliederung ein Ansatz zugrunde, dem- 
zufolge die Strukturbedingungen einer Gesellschaft erschlossen werden über die Analyse und Kritik ihrer Kategorien. Entsprechend wird vorwiegend anhand der theoretischen Primärschriften aus der Durchsetzungsperiode der bürgerlichen Gesellschaft gearbeitet werden.

Die Auswahl der ersten vier Unterpunkte ergibt sich für I-3 systematisch aus konstitutiven Merkmalen der bürgerlichen Gesellschaft. Punkt 4 gilt dessen historischer Entfaltung im Industrialisierungsproxeß.

Die ersten vier Veranstaltungsteile werden etwa das erste Studienjahr in Anspruch nehmen. In den fakultativen Arbeitsgruppen soll auf Grundlage der bis dahin in den Veranstaltungen VAK 40 bis VAK $4 \mathrm{I}$ erarbeiteten Kategorien mit der Projektvorbereitung begonnen werden. Der Veranstaltungsteil s soll die Enthistorisierung und einzelwissenschaftliche Differenzierung innerhalb der Sozialwissenschaften behandeln. Die Ausgestaltung erfolgt im Zusammenhang mit der Arbeit im ersten Studienjahr.

S.3 Arbeitskampf in der Bundesrepublik

An der Planung beteiligte Hochschullehrer: Hoffmann, Steinberg, Billerbeck, Rinken, Lautmann, Hinz, Biesecker, Wohlmuth, Preuß, Saurien, Dubischar (VAK 42 )

Problematisierung eines Arbeitskampfes (Streik bzw. Mitbestimmungskonflikt)

A. Kapital- und Einkommensverteilung in der BRD/Aktuelle Probleme der Einkommenspolitik

a) Entwicklung der Produktivkräfte und Produktionsverhältnisse in der BRD - u. a. Wachstum, technischer Fortschritt und Produktivitätsentwicklung, Konzentration, internationale Beziehungen

b) Anpassung der Steuerungsmechanismen an die Entwicklung der Produktivkräfte - u. a. Globalsteuerung, Konzertierte Aktion

c) Einkommens- und Vermögensverteilung - im Zusammenhang mit a) und b) - u. a. Entwicklung der Lohnquote, Tarif und Effektivlohnentwicklung (wage drift), Beschättigungsentwicklung

B. Konflikte und ihre Regelungen

a) Verrechtlichung der Arbeirsbeziehungen - u. a. Tarifverträge, Betriebsvereinbarung, Mitbestimmung, Arbeitsschutz, Entwicklung des Kollektivrechts durch das BAG

b) Arbeitsbeziehungen im Betrieb

c) Streikformen und -verläufe, Verhandlungsformen und -muster - u. a. Schlichtung, gewerkschaftliche und spontane Streiks

C. Probleme der Organisationen und ihr Verhältnis zu Interesse und Bewußtsein der Vertretenen

D. Konkrete Analyse von Arbeitskonflikten in einer Branche

\section{DOKUMENT 6}

Thesen zur Bremer Juristenausbildung

verabscbiedet vom Gründungssenat der Universität Bremen am 21. I2. 1970

I. Ausgangspunkt einer Ausbildung von Juristen darf nicht allein ein "Leitbild des Juristen in unserer Zeit « sein, sei es nun das des »fachlich geschulten, geistig selbständigen und seiner Verantwortung bewußten jungen Juristen « (Münche- 
ner Beschlüsse) oder das des gesellschaftskritischen Juristen neuen Typs (Loccum).

2. Juristenausbildung heute hat sich vielmehr zu orientieren an den Tätigkeitsfeldern wissenschaftlich Qualifizierter in den Berufsbereichen Verwaltung, Justiz und rechtliche Beratung. Sie hat dabei die Instrumentalisierung von Recht und Juristen im Dienst der herrschenden Interessen bewußt $\mathrm{zu}$ machen und $\mathrm{zu}$ ihrer Überwindung beizutragen.

3. Eine durchgehend notwendige Qualifikation für diese Funktionen ist die Fähigkeit zu theoriegeleiteten Handeln. Darunter ist weder ausschließlich der Erwerb und die Anwendung gewisser Fertigkeiten und heuristischer Verfahrensregeln zu verstehen, noch ausschließlich der Erwerb und die Anwendung wissenschaftlich analytischer Fähigkeiten.

4. Ausbildung für so verstandene gesellschaftliche Praxis heißt stattdessen: Vermittlung der im jeweiligen Praxisbereich notwendigen Techniken bei gleichzeitiger Analyse des geschichtlichen Ursprungs und der theoretischen Ableitung dieser juristischen Formen sowie der gesellschaftichen und politischen Funktion ihres Anwendens in spezifischen Situationen.

5. Damit ergeben sich einige Grundvoraussetzungen juristischer Ausbildung:

a) Die Ausbildung muß ständig gleichzeitig an der konkreten gesellschaftlichen Praxis von Juristen wie an der theoretischen Begründung dieser Praxis durch Rechtstvissenschaft und Sozialwissenschaften (i. e. S.) orientiert sein. Dies ist nur möglich in einem einphasigen, projektorientierten, die Grenzen konventioneller Fächer transzendierenden Studium.

b) Die Ausbiidung muß dabei nicht nur den Prozeß des intellektuellen Lernens sondern die gesamte Sozialisation während des Studiums in Hinblidk auf die zu erreichenden Qualifikationen reflektieren. Auch dies ist durch neue Ausbildungsformen und flexible Organisation und Inhalte sicherzustellen.

c) Die Ausbildung muß in einer Anfangsphase sowohl die Orientierung der Studenten über in Aussicht genommene gesellschaftiche Funktionen und die dafür notwendigen Qualifikationen ermöglichen, wie auch die theoretischen Fähigkeiten für die Ableitung und Einordnung der dort zu leistenden Praxis vermitteln (sozialwissenschaftliches Eingangsstudium).

d) Die Ausbildung soll in einer mittleren Phase dann intensiv auf die spezifisch juristische Funktion in unserer Gesellschaft (Gestaltung gesellschaftlicher Wirklichkeit schwerpunktmäßig in formalisierten Verfahren nach abstrakten Normen) vorbereiten. Dabei werden die Projekte nicht so sehr darauf abzielen dürfen, das gesamte Gebäude des geltenden Rechts in seiner Dogmatik zu vermitteln, als vielmehr die Erarbeitung der Grundstrukturen und Schwerpunkte rechtlicher Regelungen in praktischen gesellschaftlichen Anwendungsbereichen zu strukturieren.

e) In der Endphase werden die Studenten sich dann auf ihre juristisch spezifischen Praxisbereiche [in der Anfangsphase der Universitär: Verwaltung und Justiz, dort besonders Zivil(Arbeits)-Recht] schwerpunktmäßig vorbereiten, ohne dabei den juristischen und gesellschaftlichen Gesamtzusammenhang zu verlieren.

6. Diese Grundvoraussetzungen wird die Planungskommission Sozialwissenschaften im weiteren Planungsprozeß konkretisieren, und zwar durch Erarbeitung von

- Projektthemen und den in den Projekten zu vermittelnden Inhalten

- Evaluationsmethoden der in den Projekten vermittelten Qualifikationen (studienbegleitende Leistungskontrollen, Examen) 
- Organisationsvorschläge zum gesamten Studiengang (Verbindung von Grundstudium, Projekten, Intensivkursen etc.)

- Organisationsstrukturen des Lehr- und Forschungsbetriebes an der Universität (Projektbereiche, Fachbereiche, politischer Entscheidungsgang)

Die PKS erwartet, daß die zuständigen Bremer Instanzen bei der Erarbeitung einer JAG sowohl diese Grundvoraussetzungen wie auch die noch folgenden Konkretisierungen berücksichtigen. Sie geht insbesondere davon aus, daß keine Regelungen getroffen werden, die die Ausführung ihrer Konzepte beeinträchtigen.

Die Konkretisierungsarbeit ist keine im Planungsprozeß abschließbare, sie wird vielmehr dauernde Leistung der an der Reformuniversität Bremen Lehrenden und Lernenden sein. Dem müssen alle außeruniversitären Instanzen, die in Form von Berufseingangsvoraussetzungen, Prüfungs- und Ausbildungsverordnungen Strukturentscheidungen auch für die Universität fällen - im Falle der Juristenausbildung also der für in JAG zuständige Justizsenator - durch offene und flexible Regelungen Rechnung tragen.

\section{DOKUMENT 7}

Aus einem Bericht des für die Juristenausbildung zuständigen Planers vom 20. I. 1972 über die Studienplanung für Rechtswissenscbaften

2.1 I Die wissenschaftspolirischen Ausgangsdaten der Planung der Juristenausbildung an der Universität Bremen im Herbst 1970 waren (a) die Bremer Konzeptionen eines sozialwissenschaftlichen Eingangsstudiums für alle im Planungsbereich Sozialwissenschaften vorgesehenen Studiengänge (Frankfurter und Göttinger Papier, Kritische Justiz 1970, 327-335) sowie des Projektstudiums (Beiträge zur Studienreform, Materialien der Bundesassistentenkonferenz Bonn, 1970, Heft 7) und (b) das von den Reformkräften der westdeutschen Juristenausbildungsreformdiskussion der letzten Jahre entwickelte Konzept der einstufigen Juristenausbildung. Dieses sieht eine Verbindung von Studium/»Theorie « und juristischem Vorbereitungsdienst/»Praxis« zu einem Ausbildungsgang, eine Integration der Juristenausbildung in die Sozialwissenschaften und eine Reform des Prüfungswesens vor (Neue Juristenausbildung, Materialien des Loccumer Arbeitskreises zur Reform der Juristenausbildung, Neuwied-Berlin 1970).

Diese Rahmenbestimmungen wurden in der Folgezeit durch verschiedene weitere Diskussionsvorschläge zum sozialwissenschaftlichen Eingangsstudium von Mitgliedern der Planungskommission Sozialwissenschaften (PKS) und durch die Entscheidung des Gründungssenats der Universität Bremen (GS) vom 21. 3. 1971 konkretisiert, das Studium der Rechtswissenschaften, Wirtschaftswissenschaften und Sozialwissenschaften i. e. S. im Wintersemester $1971 / 72$ mit einem integrierten Eingangsstudium beginnen zu lassen. Ferner entwickelte die Unterkommission Jura (UKJ) entsprechend den Gründungssenatsbeschlüssen vom 19. I2. 1970 zum Projektstudium und vom 2I. I2. I970 zu den "Thesen zur Bremer Juristenausbildung * (vervielf.) zu Beginn des Jahres 1971 mehrere Vorschläge für "Projekte im Bereich der Juristenausbildung « (vervielf.).

Auf der Grundlage von Vorschlägen eines Arbeitsausschusses der PKS zur Vorbereitung des sozialwissenschaftlichen Eingangsstudiums, der in der 
Sommerpause tagte, und eines »Gutachtens zum integrierten sozialwissenschaftlichen Eingangsstudium an der Universität Bremen unter besonderer Berücksichtigung des Frankfurter Reformversuchs mit Studienanfängern an der juristischen Fakultät« von G. Bechmann, Dr. H.-J. Koch und H. Rottenleuthner (Frankfurt, August 1971, vervielf.) begann die Arbeitsaufnahme zwischen Hochschullehrern und Tutoren im Bereich Sozialwissenschaften und PKS. Kurz vor Semesterbeginn einigte man sich auf ein Konzept für das erste (und z. T. schon das zweite) Semester des Eingangsstudiums (Studienführer und Personalverzeichnis der Universität Bremen WS 1971/72, 10I-106; Zwischenbericht über Stand und Weiterplanung der Veranstaltungen des sozialwissenschaftlichen Eingangsstudiums vom 7. I. 1972).

2.I 2 Die Bemühungen der Universität Bremen um die Einführung einer reformierten Juristenausbildung in Bremen wurden durch einen 1970 verabschiedeten Entwurf der Bundesregierung zur Änderung des Deutschen Richtergesetzes (DRiG) und eine darin enthaltene Klausel zur Erprobung einer einstufigen Juristenausbildung ermöglicht (BT-Drucksache VI/I 380 ). Am 19. 9. I 971 verabschiedete der Deutsche Bundestag die Experimentierklausel in einer abgewandelten Fassung (BGBl. I/1971, Nr. 96, I 557I 560 ).

Der GS hatte mit seinen "Thesen zur Bremer Juristenausbildung " schon frühzeitig seinen Wunsch erklärt, daß in Bremen eine Juristenausbildung nach der Experimentierklausel eingeführt wird. Der bremische Senator für Justiz und Verfassung reagierte darauf am i 8 . I. I 97 I mit der schriftlichen Erklärung, daß die Experimentierklausel nach ihrem Inkraftreten einer gründlich vorbereiteten Konkretisierung durch das Landesrecht bedürfe und dazu die Einsetzung einer Kommission aus Vertretern der Universität und der Praxis erforderlich sei.

Dies geschah am 25.2. I971 durch die Bildung eines Unterausschusses der Fachdeputation; dieser setzte die Kommission ein und gab ihr den Auftrag, sie solle »... ein Modell für die künftige Juristenausbildung in Bremen erarbeiten, auf seine Durchführbarkeit überprüfen und ein Juristenausbildungsgesetz entwerfen « (Mitteilungen der Pressestelle des Senats vom 26. 2. I971, 363).

$\mathrm{Da}$ in dieser Kommission die Universität nur mit einem Vertreter, die juristischen Berufe jedoch mit zehn (mit Einschluß eines Vertreters der Vereinigung Bremischer Gerichtsreferendare) repräsentiert waren, bat der GS am I8. 4. 197 I mit einer ausführlichen Begründung um halbparitätische Umgestaltung der Sachverständigenkommission. Die Deputation nahm daraufhin am 27.5.197 I vier weitere Vertreter der Universität in die Kommission auf. Der GS akzeptierte diese - drittelparitätische - Beteiligung am I 4. 6. I 97 I und benannte die fünf Universitätsvertreter.

Die Kommissionsarbeit begann am 30. 4., I./2. 5. 1971 mit einer Übersicht und Diskussion über Planungsgrundsätze, -stand und -yorhaben zur Juristenausbildung an der Universität Bremen und über die in anderen Ländern vorgeschlagenen Modelle einer einstufigen Juristenausbildung.

Zur Sitzung am 26.6. 197 I wurde von Vertretern der Justiz eine Skizze als Ablauf und Inhalt einer einstufigen Juristenausbildung als Grundlage für die weiteren Kommissionsberatungen vorgelegt. Sie beantragten zugleich, diese Weiterarbeit in Arbeitsgruppen über die Themenkreise Einbeziehung der Sozialwissenschaften in die Juristenausbildung, Verklam- 
merung von theoretischer und praktischer Ausbildung, Juristischer Stoffplan, Prüfungswesen, Projektstudium und Schwerpunktausbildung zu verlegen.

Die Universitätsvertreter setzten jedoch als »Arbeitszwischenschritt « eine wechsel seitige Orientierung über die grundlegenden Fragen einer Juristenausbildungsreform und eines angemessenen Juristenausbildungsgesetzes (JAG) durch; man einigte sich zugleich, einen Entwurf für ein bremisches JAG bis Mitte 1972 fertigzustellen.

Die zur Sitzung am 26.7.1971 von seiten der Universität vorgelegte grundsätzliche Stellungnahme (Zum Selbstverständnis der Kommission, Zum Verhältnis von Theorie und Praxis, Zum Verhältnis von Rechtsund Sozialwissenschaften, Zum Leitbild der Juristenausbildung), der ein Aufsatz ihres Verfassers Prof. Wiethölter über Didaktik und Rechtswissenschaft (in: Neue Juristenausbildung, a.a. O., 25-4I) und das Protokoll seiner Anhörung vor dem Rechtsausschuß des Deutschen Bundestages am 25.3. 197 I (Protokoll Nr. 42, 107-II4) beigefügt waren, stießen bei den Vertretern der Praxis auf mehr oder weniger verhüllte Ablehnung gegen eine derartige Vorphase der Ausarbeitung eines JAG. Die schon mehrfach vorgetragene Kritik an der mangelnden Konkretisierung des angestrebten Studienganges spitzte sich im Sommer 197 I zu einer Beratung der Präsidenten der obersten bremischen Gerichte und des Generaistaatsanwalts über eine gemeinsame Intervention gegen den Beginn der Juristenausbildung an der Universität im WS 1971/72 und in der letzten Sitzung der bremischen Bürgerschaft vor der Neuwahl am 10. 10. 1971 zu einer Anfrage der Fraktionen der FDP und der CDU zur Vereinbarkeit des - die Anfangsphase der Juristenausbildung einschließenden - sozialwissenschaftlichen Eingangsstudiums mit der bundes- und landesrechtlichen Situation zu.

Die Universitätsvertreter in der Sachverständigenkommission begründeten daher in deren Sitzungen am 16.7. und 13.8. 197 I unter teilweiser Ubernahme der Argumentation des in der $Z$ wischenzeit fertiggestellten »Gutachtens zum integrierten sozialwissenschaftlichen Eingangsstudium...* (s. o.) ausführlich die Einbeziehung der Juristenausbildung in das Eingangsstudium. Die von einer Gruppe von Praktikern und den Universitätsvertretern daraufhin angestrebte Entscheidung wurde jedoch von einem noch zögernden Teil der Kommission und seinem oppositionellen Kern von dem Ergebnis einer Anhörung dreier weiterer Sachverständiger abhängig gemacht.

Sie fand erst am 19. II. 1971 statt (Wortprotokoll vom Januar 1972); in einer anschließend von der Kommission beschlossenen Presseerklärung heißt es: „Uber das Verhältnis von Rechts- und Sozialwissenschaften im Rahmen einer reformierten Juristenausbildung und die Möglichkeit eines integrierten sozialwissenschaftlichen Eingangsstudiums hörte die Bremer Kommission zur Reform der Juristenausbildung ... die Professoren Frowein, Bielefeld, Grauhan, Bremen, und Maihofer, Bielefeld. Die auswärtigen Sachverständigen stimmten im Ergebnis darin überein, daß die von Prof. Grauhan vorgestellten Uberlegungen der Universität ein diskussionswürdiges Modell darstellten, das zwar nicht ohne Risiken sei, aber Verwirklichungschancen biete, die über Bremen hinaus von Bedeutung seien, und das daher erprobt werden sollte....

Inzwischen hatte das Ergebnis der Wahl zur Bremer Bürgerschaft das von 
der CDU eröffnete, seit dem Bruch der Koalition von der FDP unterstützte Trommelfeuer gegen die Universität beendet. Mit der Bildung der sozialdemokratischen Alleinregierung begann eine - bis jetzt noch nicht voll einschätzbare - neue Phase der Beziehungen zwischen Universität und Land. Immerhin brachte die Regierungserklärung vom I 5. I2. I971 die vor der Wahl von der FDP mühsam, von der SPD behutsam offengehaltene - Option für die Einführung und Erprobung der einstufigen Juristenausbildung in Bremen. Auf erneute Bitte der Universität beschloß unter Vorsitz des neuen Senators für Rechtspflege und Strafvollzug die Fachdeputation am 29. I 2. 197 I die halbparitätische Beteiligung der Universität (einschließlich des Referendarvertreters) an der Sachverständigenkommission (unter Vergrößerung auf 22 Mitglieder). Der fortbestehende Unterausschuß präzisierte den Auftrag der Kommission am 17. I 2. 1972 dahingehend, daß sie „... bis Ende Mai dieses Jahres den Entwurf eines Juristenausbildungsgesetzes für eine einstufige Juristenausbildung und den Abriß eines Ausbildungsmodells ... (vorlegen solle). Mit dem Gesetz soll ein einjähriges integriertes sozialwissenschaftliches Eingangsstudium erprobt werden ... (Weser-Kurier, I 8. I. 1972, I4).

Die Kommission nahm ihre Arbeit am 17. I. 1972 mit der Bildung von 4 Arbeitsgruppen (Sozialwissenschaftliches Eingangsstudium; Juristischer Stoffplan; Verbindung von Theorie und Praxis; Studienbegleitende Kontrollen, Abschlußverfahren, Ausbildungs- und Prüfungsamt) und der Vereinbarung eines weiteren Hearings über $»$ Projektstudium in der Juristenausbildung " wieder auf.

2.I 3 Am 14. I. 1972 hatte sich die - bis auf 2 Vertreter der PKS - aus Vertretern der Hochschullehrer und Studenten der Universität Bremen gebildete Studiengangskommission Rechtswissenschaften konstituiert; sie arbeitet mit den Vertretern der Universität in der Landeskommission engstens zusammen.

In Anlehnung an die - schon erwarteten - Themengruppen der Landeskommissionsarbeit hat die Studiengangskommission eigene Arbeitsgruppen gebildet. Diese entwickeln zur Zeit - koordiniert durch das Plenum - ein Rahmen-curriculum für eine projektorientierte Ausbildung für Juristen im Rahmen der geplanten - ebenfalls projektbezogenen - Studienbereichsstruktur der Universität, das der im bremJAG zu konkretisierenden Experimentierklausel des DRiG genügt.

\section{Das Bundesausbildungsförderungsgesetz: Förderung der Ausbildung oder der Ausbeutung?}

Der Ausbildungssektor befindet sich heute in einer Krise, wer wüßte das nicht. Selbst der illustrierte Blätterwald bejammert die mangelnde Repräsentation der unteren Klassen an Hochschulen, höheren Schulen und ähnlichen Bildungseinrichtungen. Die Stunde der Reformen schlägt, es müssen Gesetze geschaffen werden, die die gesellschaftliche Misere beseitigen, sonst beseitigt etwa noch die gesell- 